\title{
IMPACTO DE LA CONTAMINACIÓN ATMOSFÉRICA Y EL CLIMA EN LAS CONSULTAS A UN DEPARTAMENTO DE EMERGENCIAS PEDIÁTRICO EN LA CIUDAD DE BUENOS AIRES
}

\author{
IMPACT OF AIR POLLUTION AND CLIMATE ON A PEDIATRIC EMERGENCY DEPARTMENT VISITS IN \\ BUENOS AIRES CITY
}

Mg. Rosana Abrutzky ${ }^{1,2}$, Dr. Fernando Adrián Torres ${ }^{3}$, Dra. María Fabiana Ossorio ${ }^{3}$, Dr. Fernando Ferrero ${ }^{4}$

\section{Resumen:}

Objetivo: evaluar el impacto de los contaminantes atmosféricos y el clima en el número de consultas al departamento de emergencias de un hospital pediátrico en Buenos Aires, Argentina. Material y métodos: estudio ecológico utilizando análisis de series temporales con modelos aditivos generalizados (GAM) para el período 2012-2014, analizando cantidad diaria de consultas en función de tres contaminantes del aire: monóxido de carbono $(\mathrm{CO})$, dióxido de nitrógeno $\left(\mathrm{NO}_{2}\right)$ y material particulado de diámetro menor a 10 micrones $\left(\mathrm{PM}_{10}\right)$, temperatura diaria, humedad relativa, dirección y velocidad del viento y lluvia, controlando por día de la semana y tendencia de largo plazo. Resultados: las consultas en invierno (286 diarias) fueron significativamente mayores que en las demás estaciones $(p<0.0001)$. La variación en el nivel de contaminación del aire no mostró correlación significativa y consistente con las consultas. La temperatura, humedad relativa, lluvia y viento no resultaron significativas en ninguno de los rezagos analizados. Conclusiones: en esta población y considerando las consultas de emergencia, la contaminación del aire no afecta significativamente el resultado en salud, pero sí lo hacen las epidemias estacionales. Estudios sobre otras variables de salud son necesarios para establecer el impacto de la contaminación del aire sobre la salud infantil en Buenos Aires.

Palabras clave: Contaminación del aire; Clima; Medioambiente y salud pública

\section{Abstract:}

Objective: to evaluate the impact of air pollution and weather changes on the number of visits to a pediatric emergency department in Buenos Aires, Argentina. Materials and methods: we designed an ecological time-series study using generalized additive models (GAM) for the period 2012-2014. The outcome variable was the number of daily visits to a pediatric emergency department taking into account daily consultations. The potential predictors were the average daily air pollution levels (carbon monoxide -CO-, nitrogen dioxide $-\mathrm{NO}_{2}-$, particulate matter 10 microns or less in diameter $-\mathrm{PM}_{10^{-}}$) and weather variables (temperature, humidity, wind speed and direction, and rainfall) and we controlled by weekday and longtime trend. Results: the number of visits during the winter months ( 286 daily) was significantly higher than those in the other seasons $(p<0.0001)$. The change in air pollution levels did not show a significant and consistent correlation with the visits. Temperature, relative humidity, rainfall, and wind speed and direction were not significant variables for any of the examined lags. Conclusions: Considering the population and variables of our study, air pollution does not affect the health outcomes, as does seasonal epidemics. Studies that examine other health variables are necessary to establish the impact of air pollution on children's health in Buenos Aires.

Key words: Air Pollution; Climate; Environment and Public Health

\footnotetext{
${ }^{1}$ Universidad de Buenos Aires, Facultad de Ciencias Sociales, Instituto de Investigaciones Gino Germani

2 Email de contacto: rabrutzky@gmail.com

${ }^{3}$ Docencia e Investigación, Hospital General de Niños Pedro de Elizalde, Buenos Aires

${ }^{4}$ Departamento de Medicina, Hospital General de Niños Pedro de Elizalde, Buenos Aires
} 


\section{Introducción}

La exposición a contaminantes ambientales en zonas urbanas tiene impacto en la salud, y su control constituye uno de los mayores desafíos sanitarios a nivel mundial ${ }^{(1,2)}$.

Aún en ciudades cuyos niveles de contaminación del aire no sobrepasan los estándares aceptados, se ha constatado que el aumento en los niveles de contaminantes correlaciona con incremento en la mortalidad, las consultas a departamentos de emergencia y las hospitalizaciones, tanto en la población en general como en grupos vulnerables ${ }^{(2)}$. Más aún, se ha verificado correlación entre variaciones en un rango no extremo de contaminantes del aire y salud infantil, constatando el impacto de tres contaminantes clásicos (monóxido de carbono $\mathrm{CO}$-, dióxido de nitrógeno $-\mathrm{NO}_{2^{-}}$, material particulado menor a 10 micrones $-\mathrm{PM}_{10^{-}}$) sobre el número de consultas de emergencia pediátrica ${ }^{(3)}$. Para la Ciudad de Buenos Aires ha sido estudiada la correlación entre contaminantes atmosféricos y mortalidad ${ }^{(4,5)}$.

Por otro lado, existe evidencia sobre el impacto de factores climáticos en la salud ${ }^{(6,7)}$. En pediatría es conocido el impacto de la temperatura en la salud infantil ${ }^{(8)}$; particularmente en regiones de clima templado el invierno suele asociarse a incremento de las infecciones respiratorias, incluyendo bronquiolitis e influenza ${ }^{(9)}$.

A pesar que es conocida la influencia que el clima puede tener en los niveles de contaminación ambiental ${ }^{(10)}$, hay menos certeza sobre el impacto de la interacción entre clima y polución ambiental en la salud ${ }^{(11)}$.

Nuestro objetivo fue evaluar la evolución de las consultas en el departamento de emergencias de un hospital pediátrico y su relación con factores de clima y contaminantes del aire.

\section{Métodos}

Diseño: Estudio ecológico utilizando análisis de series temporales con Modelos Aditivos Generalizados.

Ámbito: Ciudad de Buenos Aires, Argentina.

Datos: Se construyó una serie temporal con 1095 registros diarios de todas las variables, correspondientes al período 2012-2014.

Los datos de consultas fueron provistos por la División de Estadística del Hospital General de Niños Pedro de Elizalde (HGNPE), incluyendo todos los pacientes (menores de 18 años de edad) asistidos en el departamento de emergencias en el período mencionado.

Los datos de calidad del aire provienen de la Agencia de Protección Ambiental de la Ciudad de Buenos Aires. Los contaminantes medidos fueron dióxido de nitrógeno $\left(\mathrm{NO}_{2}\right)$, monóxido de carbono (CO) y material particulado de diámetro menor a 10 micrones $\left(\mathrm{PM}_{10}\right)$. Los niveles fueron registrados por tres estaciones de monitoreo automáticas ubicadas en diferentes zonas de la ciudad: Parque Centenario (zona residencial), Córdoba (zona de tránsito vehicular intenso), y La Boca (zona urbana fabril). Se utilizaron promedios de 24 horas.

Los datos meteorológicos fueron provistos por el Servicio Meteorológico Nacional, tomados de la estación Central (Villa Ortúzar). La Ciudad de Buenos Aires tiene clima templado, con cuatro estaciones bien diferenciadas, que para el presente trabajo fueron delimitadas con los parámetros del Servicio Meteorológico Nacional (otoño: marzo, abril y mayo; invierno: junio, julio y agosto; primavera: septiembre, octubre y noviembre; verano: diciembre, enero y febrero ${ }^{(12)}$.

Tratamiento de datos faltantes: Las bases de contaminantes presentaron datos faltantes (2.916 de 9.864). Para completarlas se utilizó un procedimiento de regresión lineal construido sobre promedios de observaciones temporalmente similares ${ }^{(13,14)}$. Se completó la serie de CO con el promedio del mismo día de la semana del mismo mes de los años anterior y posterior. A partir de esa serie completa se aplicó una regresión lineal simple a la serie de $\mathrm{NO}_{2}$, completando los valores con los predichos en la regresión. El mismo procedimiento se aplicó a la serie de PM10 utilizando una regresión bivariada de las series de $\mathrm{CO}$ y NO $\mathrm{N}_{2}$. Para este análisis se utilizó IBM SPSS Statistics 20.

Modelo Aditivo Generalizado: Asumiendo que la relación entre salud y polución es lineal, y la existente entre salud y variables meteorológicas no lo es ${ }^{(15)}$, utilizamos modelos no paramétricos, construyendo Modelos Aditivos Generalizados para estimar la relación entre la respuesta y los predictores $^{(16)}$. Dada la sobredispersión de los datos, los modelos se corrieron con un método de estimación quasi máximo verosimilitud o quasi-Poisson.

Se utilizó el protocolo APHEA ${ }^{(15)}$ como guía para el análisis. En la primera etapa se incluyeron día de la semana (como variable binaria), tendencia de largo plazo (spline de suavizado simple) y estacionalidad (spline cúbico cíclico). En la segunda etapa se introdujeron las variables 
meteorológicas, incluyendo rezagos de 1 a 5 días antes y rezagos de múltiples días, incorporando las medias móviles del mismo día y los 1 a 5 días previos $^{(17)}$. Finalmente se introdujeron en el modelo las variables de contaminación.

Para la corrida de modelos se utilizó el paquete "mgcv" del software R ( $R$ Development Core Team, Vienna, Austria). La significancia estadística se definió como $p<0.05$.
Los resultados son expresados como Riesgo Relativo (RR) con sus Intervalos de Confianza del 95\% (IC95\%).

Aspectos éticos: Se solicitó y obtuvo autorización de los comités institucionales. El estudio fue registrado en el Registro Público de Proyectos de Investigación del Gobierno de la Ciudad de Buenos Aires ( $N^{\circ}$ 642/15)

\section{Resultados}

Durante el período estudiado las temperaturas se mantuvieron entre 0,1 y $39 \stackrel{\circ}{ } \mathrm{C}$, con una media de $18,2^{\circ} \mathrm{C}$. La tabla 1 muestra los valores estadísticos de las variables del estudio.

Tabla 1: Consultas diarias, contaminantes y parámetros meteorológicos durante el período de análisis. Valores diarios.

\begin{tabular}{|c|c|c|c|c|c|c|c|}
\hline & \multirow{2}{*}{ Media } & \multirow{2}{*}{ Desv. Std. } & \multirow{2}{*}{ Máximo } & \multirow{2}{*}{ Mínimo } & \multicolumn{3}{|c|}{ Percentil } \\
\hline & & & & & 25 & 50 & 75 \\
\hline Consultas (N) & 242,03 & 62,87 & 480 & 60 & 199 & 230 & 281,25 \\
\hline CO (ppb) & 577,49 & 222,78 & 2600 & 100 & 400 & 514 & 700 \\
\hline $\mathrm{NO}^{2}(\mathrm{ppb})$ & 18,39 & 5,81 & 55,99 & 5 & 15 & 18 & 22 \\
\hline $\mathrm{PM}_{10}\left(\mu \mathrm{g} / \mathrm{m}^{3}\right)$ & 29,74 & 11,20 & 105 & 7 & 22 & 27,96 & 35 \\
\hline Temperatura Max $\left({ }^{\circ} \mathrm{C}\right)$ & 23,35 & 6,24 & 39 & 7,4 & 18,5 & 23,5 & 28 \\
\hline Temperatura Med $\left({ }^{\circ} \mathrm{C}\right)$ & 18,21 & 5,82 & 32,7 & 3,7 & 14,1 & 18,4 & 22,5 \\
\hline Temperatura Min $\left({ }^{\circ} \mathrm{C}\right)$ & 13,86 & 5,69 & 28,2 & 0,1 & 10 & 14,1 & 18,2 \\
\hline Lluvia (ml) & 4,35 & 13,79 & 159 & 0 & 0 & 0 & 0,3 \\
\hline Humedad (\%) & 72,04 & 12,48 & 100 & 39 & 63 & 72 & 81 \\
\hline $\begin{array}{l}\text { Viento dirección } \\
\text { (decagrados; } \mathrm{N}=0^{\circ} \text { ) }\end{array}$ & 19,10 & 11,65 & 36 & 0 & 9 & 18 & 32 \\
\hline Viento velocidad $(\mathrm{km} / \mathrm{h})$ & 19,88 & 11,16 & 80 & 6 & 13 & 17 & 22 \\
\hline
\end{tabular}

Fuente: elaboración propia a partir de datos de la Agencia de Protección Ambiental del Gobierno de la Ciudad de Buenos Aires y el Servicio Meteorológico Nacional

Durante el período se registraron 265.262 consultas en el departamento de urgencias del HGNPE, con un promedio de 242 (IC95\%: 239247) consultas diarias. Las consultas presentan un patrón anual, con picos máximos en el invierno (Gráfico 1), durante el cual el promedio diario llegó a 286 consultas (IC95\%: 279-294). Luego de controlar por día de la semana y tendencia de largo plazo, el número de consultas en invierno fue significativamente mayor que en las demás estaciones $(p<0.0001)$.
Gráfico 1. Evolución diaria de la temperatura y las consultas a un departamento de emergencias pediátrico, Ciudad de Buenos Aires

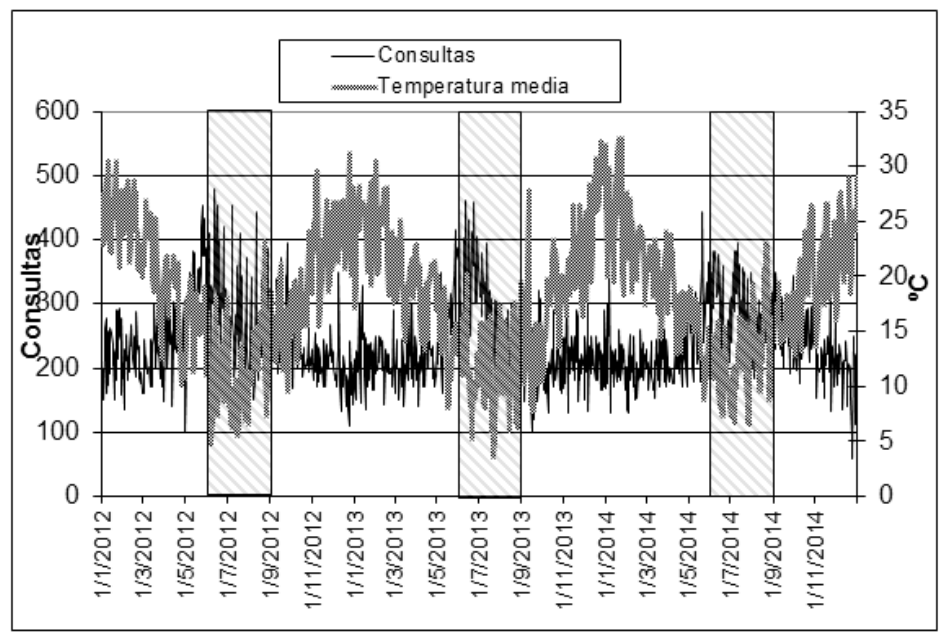

Las franjas rayadas indican los períodos invernales Fuente: elaboración propia. 
La variación en el nivel de contaminación del aire no mostró correlación significativa consistente con las consultas. Sólo para el $\mathrm{PM}_{10}$ se encontraron correlaciones negativas en algunos de los rezagos, la más importante aparece en el rezago de 3 días acumulados (promedio de mismo día y tres anteriores) (Tablas $2 a, 2 b$ y $2 c$ ). En lo referido al impacto de los contaminantes, los modelos utilizando bases parciales con temporadas de invierno y verano no mostraron diferencias con los resultados de la base completa.

La temperatura, humedad relativa, lluvia y dirección o velocidad del viento no resultaron significativas en ninguno de los rezagos analizados.

Tabla 2a: Razón de riesgo para consultas por incremento de contaminantes ambientales $\left(\mathrm{NO}_{2}: 10\right.$ ppb; CO: 100 ppb; $\left.\mathrm{PM} 10: 10 \mu \mathrm{g} / \mathrm{m}^{3}\right)$. Estación Parque Centenario

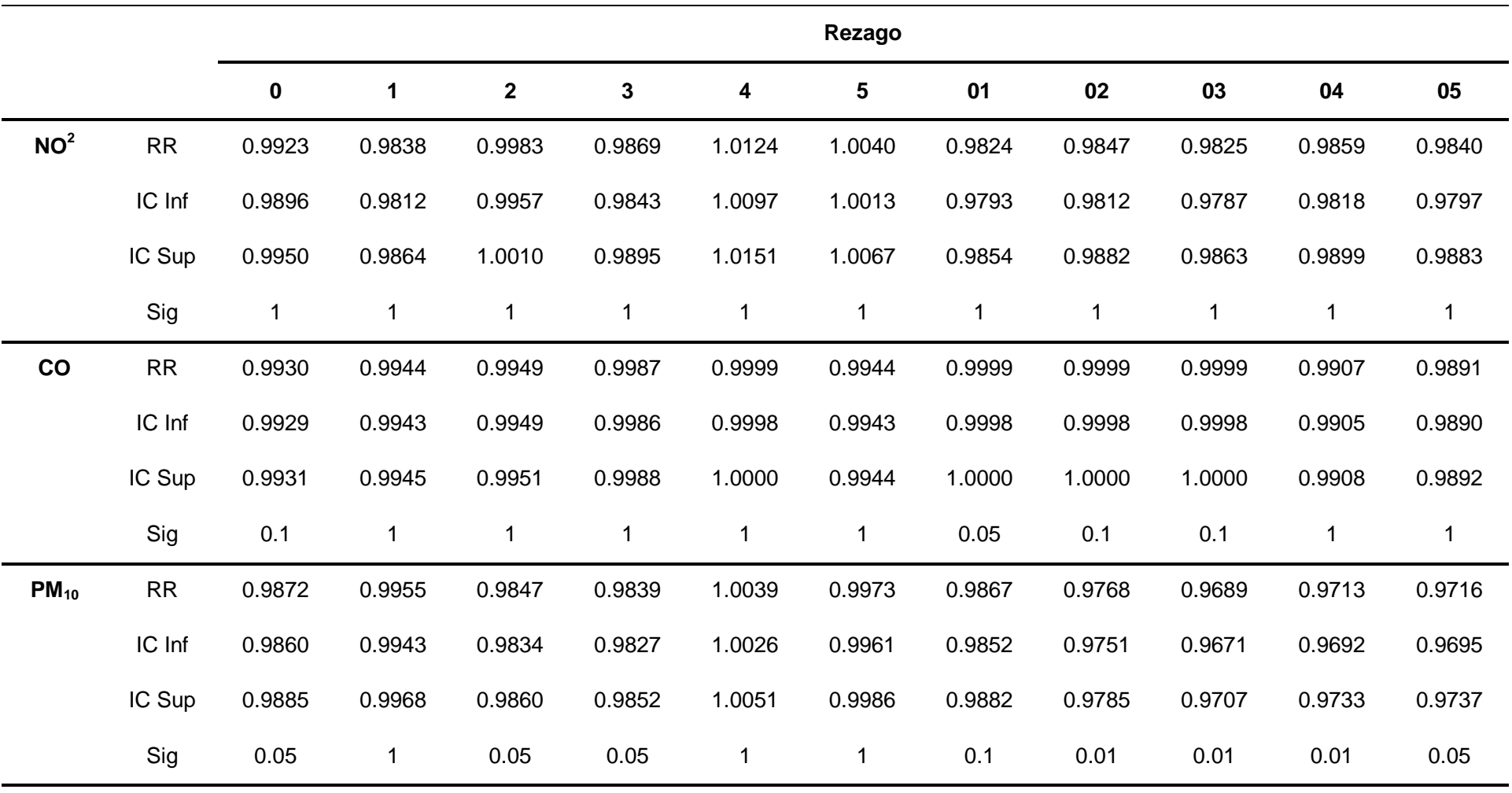

Rezagos 0 a 5: rezagos de 0 a 5 días antes

Rezagos 01 a 05: rezagos de múltiples días incorporando las medias móviles del mismo día y de los 1 a 5 días previos

Fuente: elaboración propia.

Tabla 2b: Razón de riesgo para consultas por incremento de contaminantes ambientales $\left(\mathrm{NO}_{2}: 10\right.$ ppb; CO: 100 ppb; $\mathrm{PM}$ : $\left.10 \mu \mathrm{g} / \mathrm{m} 3\right)$. Estación Córdoba

\begin{tabular}{|c|c|c|c|c|c|c|c|c|c|c|c|c|}
\hline & & \multicolumn{11}{|c|}{ Rezago } \\
\hline & & 0 & 1 & 2 & 3 & 4 & 5 & 01 & 02 & 03 & 04 & 05 \\
\hline \multirow[t]{4}{*}{$\mathrm{NO}^{2}$} & $\mathbf{R R}$ & 1,0083 & 1,0063 & 0,9991 & 1,0030 & 1,0028 & 1,0128 & 1,0086 & 1,0057 & 1,0076 & 1,0025 & 1,0071 \\
\hline & IC Inf & 1,0070 & 1,0050 & 0,9978 & 1,0017 & 1,0015 & 1,0115 & 1,0070 & 1,0040 & 1,0057 & 1,0005 & 1,0049 \\
\hline & IC Sup & 1,0096 & 1,0076 & 1,0004 & 1,0043 & 1,0041 & 1,0141 & 1,0101 & 1,0074 & 1,0095 & 1,0045 & 1,0092 \\
\hline & Sig & 1 & 1 & 1 & 1 & 1 & 0,1 & 1 & 1 & 1 & 1 & 1 \\
\hline \multirow[t]{4}{*}{ CO } & $\mathbf{R R}$ & 0,9994 & 1,0043 & 0,9993 & 1,0006 & 1,0023 & 1,0069 & 1,0052 & 1,0039 & 1,0047 & 1,0059 & 1,0084 \\
\hline & IC Inf & 0,9993 & 1,0043 & 0,9992 & 1,0005 & 1,0022 & 1,0068 & 1,0052 & 1,0038 & 1,0046 & 1,0058 & 1,0083 \\
\hline & IC Sup & 0,9996 & 1,0044 & 0,9994 & 1,0007 & 1,0024 & 1,0069 & 1,0053 & 1,0040 & 1,0049 & 1,0060 & 1,0085 \\
\hline & Sig & 1 & 1 & 1 & 1 & 1 & 0,05 & 1 & 1 & 1 & 1 & 1 \\
\hline
\end{tabular}


ARTÍCULO ORIGINAL

\begin{tabular}{|c|c|c|c|c|c|c|c|c|c|c|c|c|}
\hline \multirow[t]{4}{*}{$\mathrm{PM}_{10}$} & $\mathbf{R R}$ & 0,9936 & 1,0018 & 0,9947 & 0,9944 & 1,0081 & 1,0093 & 0,9957 & 0,9913 & 0,9848 & 0,9822 & 0,9945 \\
\hline & IC Inf & 0,9918 & 1,0000 & 0,9929 & 0,9926 & 1,0064 & 1,0075 & 0,9935 & 0,9888 & 0,9820 & 0,9793 & 0,9912 \\
\hline & IC Sup & 0,9954 & 1,0035 & 0,9964 & 0,9962 & 1,0099 & 1,0111 & 0,9978 & 0,9938 & 0,9876 & 0,9851 & 0,9977 \\
\hline & Sig & 1 & 1 & 1 & 1 & 1 & 1 & 1 & 1 & 1 & 1 & 1 \\
\hline
\end{tabular}

Rezagos 0 a 5: rezagos de 0 a 5 días antes

Rezagos 01 a 05: rezagos de múltiples días incorporando las medias móviles del mismo día y de los 1 a 5 días previos

Fuente: elaboración propia.

Tabla 2c: Razón de riesgo para consultas por incremento de contaminantes ambientales $\left(\mathrm{NO}_{2}: 10 \mathrm{ppb} ; \mathrm{CO}: 100 \mathrm{ppb} ; \mathrm{PM}_{10}: 10 \mu \mathrm{g} / \mathrm{m}^{3}\right)$. Estación La Boca

\begin{tabular}{|c|c|c|c|c|c|c|c|c|c|c|c|c|}
\hline & & \multicolumn{11}{|c|}{ Rezago } \\
\hline & & 0 & 1 & 2 & 3 & 4 & 5 & 01 & 02 & 03 & 04 & 05 \\
\hline \multirow[t]{4}{*}{$\mathrm{NO}^{2}$} & $\mathrm{RR}$ & 0,9942 & 0,9921 & 1,0014 & 0,9982 & 1,0156 & 0,9926 & 0,9929 & 0,9945 & 0,9936 & 0,9995 & 0,9974 \\
\hline & IC Inf & 0,9921 & 0,9900 & 0,9993 & 0,9961 & 1,0135 & 0,9905 & 0,9905 & 0,9918 & 0,9906 & 0,9963 & 0,9941 \\
\hline & IC Sup & 0,9963 & 0,9942 & 1,0035 & 1,0003 & 1,0177 & 0,9947 & 0,9954 & 0,9972 & 0,9965 & 1,0026 & 1,0008 \\
\hline & Sig & 1 & 1 & 1 & 1 & 1 & 1 & 1 & 1 & 1 & 1 & 1 \\
\hline \multirow[t]{4}{*}{$\mathrm{CO}$} & $\mathrm{RR}$ & 0,9994 & 0,9908 & 0,9944 & 1,0039 & 1,0071 & 0,9945 & 0,9921 & 0,9898 & 0,9939 & 0,9986 & 0,9978 \\
\hline & IC Inf & 0,9993 & 0,9907 & 0,9943 & 1,0038 & 1,0070 & 0,9944 & 0,9920 & 0,9897 & 0,9937 & 0,9984 & 0,9976 \\
\hline & IC Sup & 0,9996 & 0,9909 & 0,9946 & 1,0041 & 1,0072 & 0,9946 & 0,9923 & 0,9900 & 0,9940 & 0,9987 & 0,9980 \\
\hline & Sig & 1 & 0,1 & 1 & 1 & 1 & 1 & 1 & 1 & 1 & 1 & 1 \\
\hline \multirow[t]{4}{*}{$\mathbf{P M}_{10}$} & $\mathrm{RR}$ & 0,9928 & 0,9915 & 0,9896 & 0,9833 & 1,0050 & 0,9968 & 0,9874 & 0,9815 & 0,9689 & 0,9772 & 0,9790 \\
\hline & IC Inf & 0,9914 & 0,9901 & 0,9881 & 0,9819 & 1,0036 & 0,9953 & 0,9857 & 0,9794 & 0,9666 & 0,9748 & 0,9764 \\
\hline & IC Sup & 0,9943 & 0,9929 & 0,9910 & 0,9848 & 1,0064 & 0,9982 & 0,9892 & 0,9835 & 0,9711 & 0,9797 & 0,9815 \\
\hline & Sig & 1 & 1 & 1 & 0,05 & 1 & 1 & 1 & 0,1 & 0,01 & 0,1 & 1 \\
\hline
\end{tabular}

Rezagos 0 a 5: rezagos de 0 a 5 días antes

Rezagos 01 a 05: rezagos de múltiples días incorporando las medias móviles del mismo día y de los 1 a 5 días previos

Fuente: elaboración propia.

Durante los meses invernales la temperatura media impacta sobre la cantidad de consultas con signo negativo en rezagos posteriores al día 2 , y positivo en rezagos más cortos. Esto implica que un aumento de la temperatura produce un incremento en las consultas del día y del día siguiente, y una disminución a partir del 3er día (Gráfico 2). Durante los meses de verano no se verifica una correlación entre las variaciones a corto plazo en la temperatura diaria y la cantidad de consultas.
Gráfico 2. Riesgo (RR) de incrementar consultas por cada aumento de $1^{\circ} \mathrm{C}$ en la temperatura mínima en invierno, en los diferentes rezagos

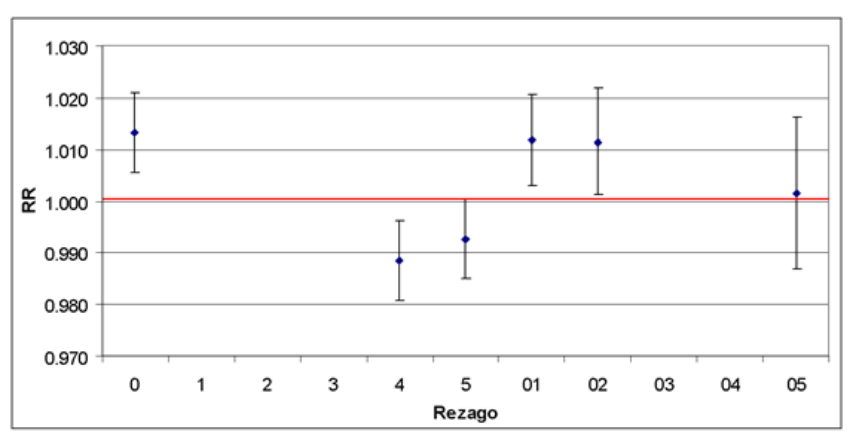

Se muestran solamente las $R R$ con significancia $<0.1$ La línea sobre $R R=1$ marca la inexistencia de asociación. Rezagos 0 a 5: rezagos de 0 a 5 días antes

Rezagos 01 a 05: rezagos de múltiples días incorporando las medias móviles del mismo día y de los 1 a 5 días previos

Fuente: elaboración propia. 


\section{Discusión}

En nuestro estudio, el nivel de los contaminantes del aire no mostró relación con el número de consultas a un departamento de urgencias de un hospital pediátrico. Sin embargo, la estación del año (invierno) se asoció significativamente con el incremento en las consultas.

La relación entre contaminación del aire y enfermedad es constantemente estudiada, y existe abundante información sobre las formas que puede adoptar ${ }^{(18)}$. Particularmente en relación a enfermedades respiratorias y grupos de riesgo, el impacto de la polución del aire en la salud ha sido bien documentado ${ }^{(19)}$.

$\mathrm{Si}$ bien en nuestro estudio no registramos las causas de las consultas, probablemente una proporción mayoritaria de ellas era de causa respiratoria ${ }^{(20,21)}$. A pesar que existe cierta evidencia que vincula las consultas pediátricas por infección respiratoria aguda con incrementos en la polución ${ }^{(19,22)}$, incluso específicamente bronquiolitis ${ }^{(23)}$, nosotros no pudimos demostrar esa relación con el número de consultas a un departamento de urgencias.

Nosotros encontramos que el número de consultas se incrementó significativamente en invierno. Es muy probable que ello se deba al incremento en las infecciones respiratorias en esa estación. Las infecciones respiratorias agudas son la principal causa de consulta en los departamentos de urgencias de los hospitales pediátricos, siendo el Virus Sincicial Respiratorio (VSR) la causa más frecuente ${ }^{(24)}$. EI VSR es también el principal responsable de hospitalización en niños ${ }^{(25)}$, estimándose puede llegar a ocasionar anualmente 40 hospitalizaciones por cada 1000 menores de un $a^{n}{ }^{(26)}$. Es conocida la relación entre el patrón de circulación del VSR y algunos determinantes climáticos. En ciudades de clima templado, como Buenos Aires, el VSR circula en invierno ${ }^{(27)}$.

En nuestro estudio no encontramos una interacción entre clima y polución con impacto en la salud. Es posible que en ello hayan coincidido dos elementos. Por un lado, la Ciudad de Buenos Aires está ubicada en una llanura sobre el estuario de un ancho río (Río de La Plata), y sujeta a vientos que soplan todo el año. Estas condiciones podrían favorecer la rápida dispersión de los contaminantes y mantener concentraciones diarias relativamente bajas, generalmente por debajo de los límites establecidos por las normas locales ${ }^{(28)}$, a pesar que las emisiones provenientes de la industria, fuentes domiciliarias y tráfico automotor en ocasiones superan las de países industrializados ${ }^{(29,30)}$. Por otro lado, como ya mencionamos, en ciudades de clima templado e inviernos definidos, el peso de la circulación viral (especialmente del VSR) en las consultas y hospitalizaciones infantiles es lo suficientemente importante como para prevalecer sobre cualquier otro determinante ${ }^{(24)}$.

El trabajo tiene las limitaciones propias de un estudio ecológico ${ }^{(31)}$, incluyendo la referida a la posible diferencia entre los sujetos expuestos al factor de análisis y aquellos que presentan el efecto. Sin embargo, el trabajo incluye los datos de uno de los tres hospitales pediátricos de la Ciudad de Buenos Aires, que asiste a niños de toda el área metropolitana sin limitaciones al acceso.

También se debe tener en cuenta que las diferentes estaciones del año, junto a sus características climáticas y meteorológicas, implican patrones de actividad, de circulación de vectores, y otras variables temporales de las cuales este estudio no da cuenta. Es así que la modalidad de agrupar los días feriados junto con los de fin de semana para fomentar el turismo utilizada por el gobierno (en 2014, 10 fines de semana fueron precedidos o seguidos de un día feriado) podría explicar la correlación negativa de las consultas con el rezago de 3 días acumulados para PM10: luego de días de tráfico intenso se suceden días feriados con mucho menor actividad. De igual forma, la restricción a la movilidad humana, especialmente la de niños pequeños, relacionada con bajas temperaturas en invierno podría explicar que el aumento de la temperatura en esa estación se correlacione con un aumento de las consultas ${ }^{(32)}$.

Finalmente, considerando que una parte de nuestros resultados podrían estar relacionados con la patología respiratoria, sería conveniente efectuar un análisis que evalúe el comportamiento de las variables al considerar exclusivamente consultas $\mathrm{u}$ hospitalizaciones relacionadas con patología respiratoria aguda.

Nuestros hallazgos refuerzan la evidencia sobre la asociación, en ámbitos urbanos de clima templado, entre el invierno y el aumento de las consultas pediátricas. Dada su naturaleza habitualmente epidémica, es importante que las autoridades sanitarias valoren adecuadamente este fenómeno para enfrentarlo convenientemente.

\section{Conclusión}

En nuestro estudio, el número de consultas en el departamento de emergencias de un hospital 
pediátrico no mostró correlación con los niveles de contaminantes en el aire. El número de consultas se incrementó significativamente en invierno.

\section{Bibliografía}

1. Ostro B, Prüss-Üstün A, Campbell-Lendrum D, Corvalán $C$, Woodward A. Outdoor air pollution Assessing the environmental burden of disease at national and local levels WHO Library Cataloguing-in-Publication Data. Geneve; 2004.

2. Pruss-Ustun $A$, Corvalan C. PREVENTING DISEASE THROUGH HEALTHY ENVIROMENTS. Towards an estimate of the enviromental burden of disease. World Heal Organ. 2006;12(2):1-106.

3. Bono R, Romanazzi V, Bellisario V, Tassinari R, Trucco G, Urbino $A$, et al. Air pollution, aeroallergens and admissions to pediatric emergency room for respiratory reasons in Turin, northwestern Italy. BMC Public Health. 2016 Dec 5;16(1):722.

4. Abrutzky $R$, Dawidowski L, Matus $P$, Lankao PR. Health Effects of Climate and Air Pollution in Buenos Aires: A First Time Series Analysis *. J Environ Prot (Irvine, Calif). 2012;3:262-71.

5. Abrutzky $R$, Ibarra $S$, Matus $P$, Lankao $P R$, Pereyra $V$, Dawidowski L. Atmospheric pollution and mortality. $A$ comparative study between two Latin American cities: Buenos Aires (Argentina) and Santiago (Chile). Int J Environ Heal. 2013;6(4):363.

6. Bernstein AS, Rice MB. Lungs in a warming world: climate change and respiratory health. Chest. 2013 May;143(5):14559.

7. du Prel J, Puppe W, Gröndahl B, Knuf M, Weigl JAl, Schaaff $F$, et al. Are Meteorological Parameters Associated with Acute Respiratory Tract Infections? Clin Infect Dis. 2009 Sep 15;49(6):861-8.

8. Xu Z, Etzel RA, Su H, Huang C, Guo Y, Tong S. Impact of ambient temperature on children's health: a systematic review. Environ Res. 2012 Aug;117:120-31.

9. Bloom-Feshbach $K$, Alonso WJ, Charu V, Tamerius J, Simonsen L, Miller MA, et al. Latitudinal variations in seasonal activity of influenza and respiratory syncytial virus (RSV): a global comparative review. Cowling BJ, editor. PLOS One. 2013 Feb 14;8(2):e54445.

10. Largeron $Y$, Staquet $C$. Persistent inversion dynamics and wintertime PM10 air pollution in Alpine valleys. Atmos Environ. 2016;135:92-108.

11. Nawrot TS, Torfs R, Fierens F, De Henauw S, Hoet PH, Van Kersschaever $G$, et al. Stronger associations between daily mortality and fine particulate air pollution in summer than in winter: evidence from a heavily polluted region in western Europe. J Epidemiol Community Heal. 2007 Feb 1;61(2):146-9.

12. Figuerola PI, Mazzeo NA. Urban-rural temperature differences in Buenos Aires. Int J Climatol. 1998 Dec;18(15):1709-23.

13.Sanhueza PA, Torreblanca MA, Diaz-Robles LA, Schiappacasse $L N$, Silva MP, Astete TD. Particulate air pollution and health effects for cardiovascular and respiratory causes in Temuco, Chile: a wood-smoke-polluted urban area. J Air Waste Manag Assoc. 2009 Dec;59(12):1481-8.

14. Neuberger M, Moshammer $H$, Rabczenko D. Acute and subacute effects of urban air pollution on cardiopulmonary emergencies and mortality: time series studies in Austrian cities. Int J Environ Res Public Health. Multidisciplinary Digital Publishing Institute (MDPI); 2013 Oct 2;10(10):4728-51.

15. Peng RD, Dominici F, Louis TA. Model choice in time series studies of air pollution and mortality. J $R$ Stat Soc Ser $A$ (Statistics Soc. Blackwell Publishing Ltd; 2006 Mar;169(2):179-203.

16. Hastie T, Tibshirani R. Generalized additive models. Chapman \& Hall/CRC; 1999. $335 p$.

17. Lin H, Liu T, Xiao J, Zeng W, Li X, Guo L, et al. Mortality burden of ambient fine particulate air pollution in six Chinese cities: Results from the Pearl River Delta study. Environ Int. 2016 Nov;96:91-7.

18. Brunekreef $B$, Holgate ST. Air pollution and health. Lancet. 2002 Oct 19;360(9341):1233-42.
19. Kurt OK, Zhang J, Pinkerton KE. Pulmonary health effects of air pollution. Curr Opin Pulm Med. 2016 Mar;22(2):138-43.

20. Ben-Isaac E, Schrager SM, Keefer M, Chen AY. National Profile of Nonemergent Pediatric Emergency Department Visits. Pediatrics. 2010 Mar 1;125(3):454-9.

21. Dirección de Estadísticas e Información de Salud. Egresos de Establecimientos Oficiales por diagnóstico -Año 2013. Buenos Aires; 2013.

22. HEI Collaborative Working Group on Air Pollution, Poverty, and Health in Ho Chi Minh City, Le TG, Ngo L, Mehta S, Do VD, Thach TQ, et al. Effects of short-term exposure to air pollution on hospital admissions of young children for acute lower respiratory infections in Ho Chi Minh City, Vietnam. Res Rep Health Eff Inst. 2012 Jun;(169):5-72-83.

23. Karr CJ, Demers PA, Koehoorn MW, Lencar CC, Tamburic L, Brauer M. Influence of Ambient Air Pollutant Sources on Clinical Encounters for Infant Bronchiolitis. Am J Respir Crit Care Med. 2009 Nov 15;180(10):995-1001.

24. Bourgeois FT, Valim C, Wei JC, MCAdam AJ, Mandl KD. Influenza and Other Respiratory Virus-Related Emergency Department Visits Among Young Children. Pediatrics. $2006 \mathrm{Jul}$ 1;118(1):e1-8.

25. Nair $H$, Nokes DJ, Gessner BD, Dherani M, Madhi SA, Singleton RJ, et al. Global burden of acute lower respiratory infections due to respiratory syncytial virus in young children: a systematic review and meta-analysis. Lancet. 2010 May 1;375(9725):1545-55.

26. Green $C A$, Yeates $D$, Goldacre A, Sande $C$, Parslow RC, McShane $P$, et al. Admission to hospital for bronchiolitis in England: trends over five decades, geographical variation and association with perinatal characteristics and subsequent asthma. Arch Dis Child. 2016 Feb;101(2):140-6.

27. Ferrero F, Torres F, Abrutzky R, Ossorio MF, Marcos A, Ferrario $C$, et al. Seasonality of respiratory syncytial virus in Buenos Aires. Relationship with global climate change. Arch Argent Pediatr. 2016 Feb 1;114(1):52-5.

28. Bogo H, Negri RM, Román ES. Continuous measurement of gaseous pollutants in Buenos Aires city. Atmos Environ. 1999 Jul;33(16):2587-98.

29. Bogo H, Gómez DR, Reich SL, Negri RM, San Román E. Traffic pollution in a downtown site of Buenos Aires City. Atmos Environ. 2001 Apr;35(10):1717-27.

30. D’Angiola A, Dawidowski LE, Gómez DR, Osses M. On-road traffic emissions in a megacity. Atmos Environ. 2010 Feb;44(4):483-93.

31. Sedgwick P. Ecological studies: advantages and disadvantages. BMJ. 2014 May 2;348:g2979.

32. Lee HJ, Jin MH, Lee JH. The association of weather on pediatric emergency department visits in Changwon, Korea (2005-2014). Sci Total Environ. 2016 May 1;551-552:699705. 